\title{
Sgluons in the same-sign lepton searches
}

\section{Wojciech Kotlarski}

Faculty of Physics, University of Warsaw,

Pasteura 5, 02093 Warsaw, Poland

Institute for Nuclear and Particle Physics, TU Dresden

Zellescher Weg 19, 01069 Dresden, Germany

E-mail: wojciech.kotlarski@fuw.edu.pl

ABSTRACT: This work presents the interpretation of the ATLAS search of same-sign lepton production in association with $b$-jets in the context of the 4-top quark signal from sgluon decays. It is shown that using just 3.2/fb data sample from Run 2 collected in 2015 the exclusion limit is already competitive with the Run 1 limit. This data makes it possible to exclude sgluons with masses up to $0.95 \mathrm{TeV}$. Prospects for the total Run 2 integrated luminosity of $100 / \mathrm{fb}$ are briefly discussed.

Keywords: Supersymmetry Phenomenology, QCD Phenomenology

ArXIV EPRINT: 1608.00915 


\section{Contents}

1 Introduction 1

2 Description of the model 2

$3 \quad$ NLO QCD corrections to sgluon pair production 3

4 Monte Carlo simulation setup 4

4.1 Signal 6

$\begin{array}{ll}4.2 \text { Background validation } & 6\end{array}$

5 Recasting current ATLAS $13 \mathrm{TeV}$ analysis 6

6 Conclusions 10

$\begin{array}{ll}\text { A Pythia8 technical setup } & 11\end{array}$

\section{Introduction}

With the Large Hadron Collider (LHC) delivering data at an unprecedented energy of $13 \mathrm{TeV}$, much work has been devoted to their interpretation in the context of BSM physics. For the time being, the main focus is on the Minimal Supersymmetric Standard Model (MSSM), or the so-called exotics. This of course leaves a lot of interesting models out. From the viewpoint of supersymmetry, this is a serious limitation. Recent years have brought considerable attention to the extended SUSY models, from simple extensions such as the NMSSM to models with an extended QCD sector such as various models with Dirac gluinos. Studies have proved that in many cases MSSM bounds are not applicable to these models [1]. On the other hand, $13 \mathrm{TeV}$ data might already be more constraining than the 7 and $8 \mathrm{TeV}$ ones, even though the collected integrated luminosity is smaller. This raises an important question about the validity of such models in light of new data.

Especially interesting are the multi top-quark processes which, while characterized by a high mass scale, enjoy big boosts in cross sections when going from 7 or 8 to $13 \mathrm{TeV}$. The 4-top quark final state was already searched for by ATLAS [2-5] and CMS [6-8] at Run

2. In the MSSM, this kind of final state may appear as decay products of 3rd generation stops produced as decay products of intermediate gluinos. In general SUSY models, the resonance structure might be quite different, though. For example, one might expect a twobody decay of a new color resonance directly to a $t \bar{t}$ pair. This is a general feature of models containing color octet (EW-singlet) scalars, commonly known as sgluons. Their LHC phenomenology was previously investigated in the context of $R$-symmetric $/ \mathcal{N}=2 /$ Dirac gaugino SUSY models, hyper-pions in vector-like confinement gauge theories and universal extra dimensions [9-21]. 
The Minimal $R$-Symmetric Supersymmetric Standard Model (MRSSM) [22] is a particularly well motivated BSM model [23-29]. Recent analyses [24, 25] have shown at full one- and leading two-loop levels that the $125 \mathrm{GeV}$ Higgs boson can be consistently obtained in agreement with precision EW observables and flavor constraints. Moreover, interesting scenarios which provide a viable candidate for dark matter have been identified [26].

Within the framework of the MRSSM [22], sgluons are expected to decay, depending on their mass, mainly into gluons or top quarks. These kinds of signatures, in both channels, were searched for by the experimental collaborations in 7 and $8 \mathrm{TeV}$ data. ATLAS excludes at $95 \%$ CL pair produced, complex sgluons decaying (with branching ratio 1) to gluon pairs in mass range from 100 to $287 \mathrm{GeV}$ [30]. For $t \bar{t}$ decay mode, sgluons are excluded at $95 \%$ CL up to $1.06 \mathrm{TeV}$ [31]. It should be noted, however, that these exclusions are based on a simplified model with a complex sgluon from ref. [32] while in the MRSSM the cross section is roughly 2 times smaller. ${ }^{1}$ At the time of writing there are no $13 \mathrm{TeV}$ analyses directly addressing sgluon pair production. Therefore, all the mentioned exclusions come from Run 1. This makes any projections for the target Run 2 integrated luminosity very difficult. To fill this gap, this work recasts ATLAS limits from search of SUSY in the 4-top quark final state in ref. [2] to sgluon pair production.

The paper is structured as follows. The next section describes and motivates the effective sgluon model used in this work. Section 3 presents NLO cross sections for the sgluon pair production. In section 4, the setup for the Monte Carlo simulation is described. Section 5 describes the parametrization of the detector response and the encoded ATLAS analysis. The reproduced analysis is validated on the associated production of top quark pair and a gauge boson in the Standard Model (SM) and on the production of gluinos in the MSSM, comparing predicted numbers of events with ones given in the ATLAS work. The analysis is then applied to the signal events. This work finishes with the derivation of the limit on the sgluon mass and prospects for this limit for the predicted $\gtrsim 100 \mathrm{fb}^{-1}$ data sample of Run 2 .

\section{Description of the model}

In the framework of a simplified model, inspired by the MRSSM scenario in which all the superpartners but the $C P$-odd sgluon are heavy, the $\mathrm{SM}$ is extended by a real color-octet (EW-singlet) scalar $O$. It couples exclusively to gluons and top quarks as given by the Lagrangian

$$
\mathcal{L}=\mathcal{L}^{\mathrm{SM}}+\frac{1}{2} D_{\mu} O^{a} D^{\mu} O^{a}-\frac{1}{2} m_{O}^{2} O^{2}-\imath c \bar{t} \gamma^{5} T^{a} t O^{a},
$$

where $D_{\mu}$ is the $\mathrm{SU}(3)_{C}$ covariant derivative and sum over the color index $a$ is understood. This Lagrangian is motivated by the MRSSM, in which a complex sgluon field is split into $C P$-even and -odd components through a $D$-term SUSY breaking contribution [25]. ${ }^{2}$ The masses of the components are then $m_{O_{S}}^{2}=m_{O}^{2}+4\left(M_{O}^{D}\right)^{2}$ for the scalar and $m_{O_{A}}^{2}=m_{O}^{2}$ for the pseudoscalar, where $m_{O}$ and $M_{O}^{D}$ are sgluon and Dirac gluino soft masses. Since

\footnotetext{
${ }^{1}$ The ATLAS analysis also does not specify the form of the sgluon-top quark coupling.

${ }^{2}$ We neglect possible (anti-)holomorphic soft-breaking sgluons mass terms.
} 

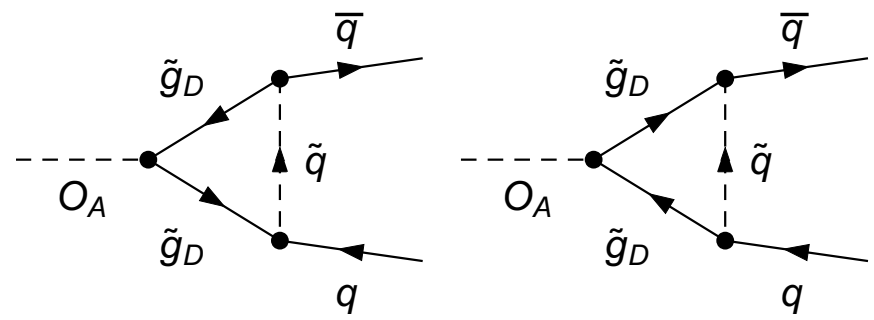

Figure 1. Lowest-order diagrams generating (effective) coupling of the pseudoscalar sgluon $O_{A}$ to quarks.

physical gluino mass, which at the tree-level is exclusively controlled by the $M_{O}^{D}$, must be $\gtrsim 1 \mathrm{TeV}$, this implies that either the pseudoscalar sgluon is very light and the scalar one is in a $\mathrm{TeV}$ range or, if pseudoscalar's mass is around $1 \mathrm{TeV}$, the scalar one will be in the multi- $\mathrm{TeV}$ range. The focus here is on the latter scenario, which extends the SM with a pseudoscalar sgluon which for simplicity's sake is denoted simply by $O$ (without the $A$ subscript).

Since in the MRSSM sgluon carries an R-charge 0, once produced it can decay to SM particles. The lowest-order coupling to quarks is loop-induced as shown in figure 1 . Coupling to gluons vanishes for pseudoscalar sgluons, while coupling to quarks is proportional to a quark mass due to chirality. Pseudoscalar sgluons with mass $m_{O_{A}} \gtrsim 2 m_{t}$ and smaller than other color-charged SUSY particles will therefore decay almost exclusively to top quarks with the coupling in the form written in eq. (2.1). Single sgluon production through (loop-induced) coupling to partons can be neglected, since it occurs mainly through coupling of gluons to the $C P$-even sgluon, which is significantly heavier than the $C P$-odd one and whose production is additionally suppressed by a small value of loop-induced coupling.

It should be noted, though, that the effective model described by the Lagrangian from eq. (2.1) is quite generic and can come from a multitude of complete, high-scale theories. However, different models would then by characterized by a different chiral structure of the coupling $c$.

\section{$3 \quad$ NLO QCD corrections to sgluon pair production}

For the Lagrangian of eq. (2.1) sgluons are produced at the LO through the Feynman diagrams in figure 2 . The corresponding partonic cross sections are: ${ }^{3}$

$$
\begin{aligned}
\hat{\sigma}_{q \bar{q}}^{B} & =\frac{2 \pi \alpha_{s}^{2}}{9 \hat{s}} \beta^{3}, \\
\hat{\sigma}_{g g}^{B} & =\frac{3 \pi \alpha_{s}^{2}}{32 \hat{s}}\left(27 \beta-17 \beta^{3}+6\left(-3+2 \beta^{2}+\beta^{4}\right) \operatorname{arctanh} \beta\right),
\end{aligned}
$$

\footnotetext{
${ }^{3}$ With an additional factor of $1 / 2$ compared to cross sections for a complex sgluon pair production considered in ref. [9].
} 


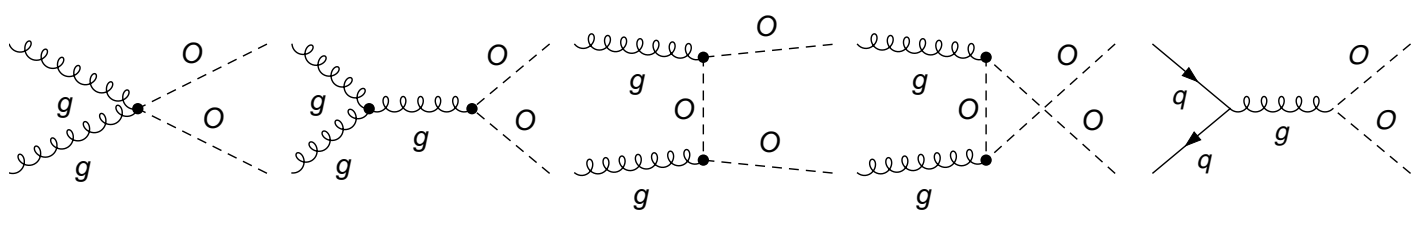

Figure 2. Feynman diagrams for the sgluon pair production at the LO.

where $\hat{s} \equiv\left(p_{q}+p_{\bar{q}}\right)^{2}$ or $\left(p_{g}+p_{g^{\prime}}\right)^{2}$ and $\beta$ is the sgluon's velocity in the center of mass system of colliding partons.

The first calculation of higher-order corrections to the sgluon pair production was done in ref. [32] for a simplified model with a complex sgluon. Since ref. [21], a general procedure for obtaining NLO-capable UFO [33] models for MadGraph5_aMC@NLO [34] using conjunction of FeynRules [35], NLOCT [36], FeynArts [37] and FormCalc [38] became available. In ref. [21] this procedure was applied to, among others, obtaining an NLO model for a real sgluon field. Since the original model used in ref. [21], available under [39], does not work for the complex coupling $\imath c$ as in eq. (2.1), a new model (this time in 5-flavor scheme) was generated and used for this analysis. ${ }^{4}$

Table 1 lists values of cross sections obtained with this model for 5 selected sgluon masses: 1, 1.25, 1.5, 1.75 and $2 \mathrm{TeV}$, for 13 and $14 \mathrm{TeV}$ LHC. Numbers were obtained using the MMTH2014 baseline (5-flavor) NLO fit (MMTH2014nlo68cl) [40] interfaced through LHAPDF6 [41]. The $K$-factors listed in the table are defined as $K \equiv \sigma_{\mathrm{NLO}} / \sigma_{\mathrm{LO}}$ and refer to the LO calculation with MMTH2014 baseline LO fit with $\alpha_{s}\left(m_{Z}\right)=0.135$ and up to 5 active flavors (MMTH2014lo68cl). For the sgluon with mass of $1 \mathrm{TeV}$, one expects more than 100 events already with the $3.2 \mathrm{fb}^{-1}$ data sample collected in 2015. Figure 3 shows the plot of the cross section as a function of the sgluon mass together with uncertainty bands for the $K$-factor coming from the PDFs (middle subplot) and the variation of renormalization/factorization scales by a factor of 2 (bottom subplot). The central values of renormalization and factorization scales are set equal to the sgluon's mass while $m_{t}=173 \mathrm{GeV}$.

Results of an automated MadGraph5_aMC@NLO calculation at the NLO were crosschecked with an independent computation based on FeynArts, FormCalc and the two-cut phase space slicing method [42]. Details of this computation are given in ref. [43].

\section{Monte Carlo simulation setup}

We now proceed to the description of methods used in the simulation of signal and background processes. Due to technical reasons, samples for signal and background were generated using two different methods outlined in the next two subsections. Both for signal and background simulation, the following values of SM gauge-boson masses were

\footnotetext{
${ }^{4}$ The NLO UFO model used for this analysis can be found in supplementary materials of the arXiv version of this work.
} 


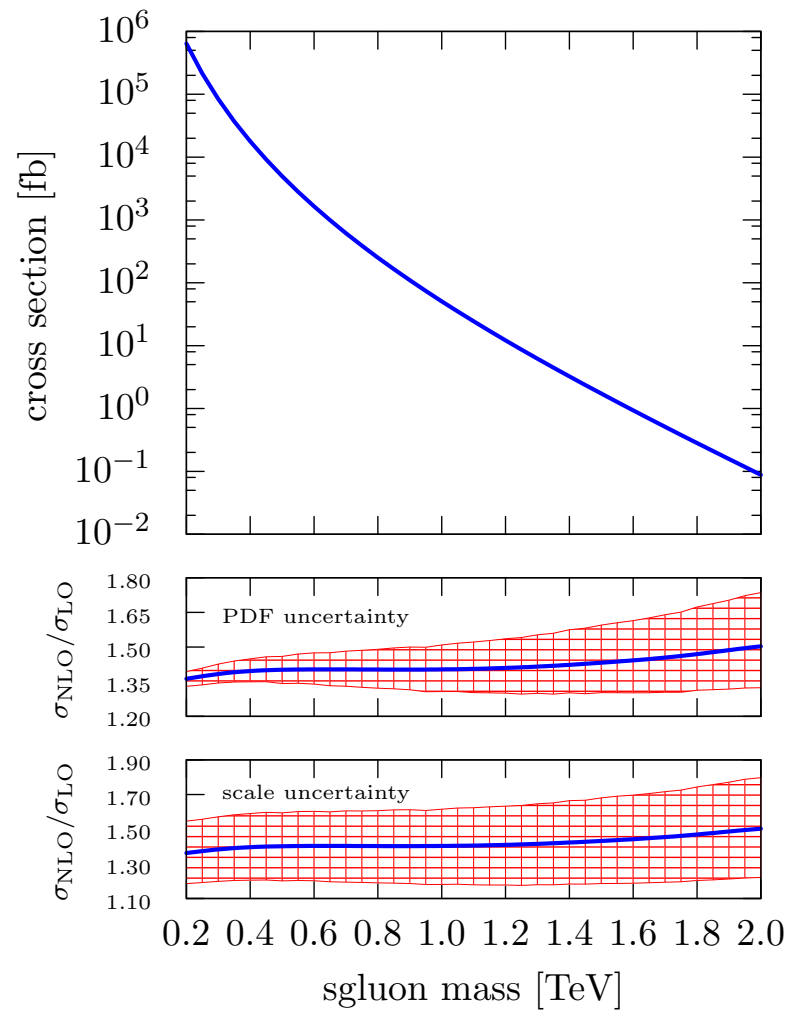

Figure 3. NLO cross section for the sgluon pair production as a function of their mass. Middle subfigure shows the $K$-factor (blue line) together with the uncertainty band coming from the PDFs. The lower one does the same for the uncertainty coming from the scale variation.

\begin{tabular}{|c|cc|cc|}
\hline sgluon mass $[\mathrm{TeV}]$ & cross section at $13 \mathrm{TeV}[\mathrm{fb}]$ & $K$ & cross section at $14 \mathrm{TeV}[\mathrm{fb}]$ & $K$ \\
\hline 1 & $50.8_{-15.7 \%-6.7 \%}^{+15.3 \%+7.7 \%}$ & 1.40 & $71.4_{-15 \%-6.3 \%}^{+14.1 \%+7.2 \%}$ & 1.37 \\
1.25 & $8.66_{-16.5 \%-7.9 \%}^{+16.3 \%+9.5 \%}$ & 1.38 & $12.9_{-15.7 \%-7.4 \%}^{+14.9 \%+8.8 \%}$ & 1.41 \\
1.5 & $1.73_{-17.2 \%-9.1 \%}^{+17.3 \%+11.3 \%}$ & 1.40 & $2.75_{-16.3 \%-8.5 \%}^{+15.8 \%+10.5 \%}$ & 1.39 \\
1.75 & $0.380_{-17.9 \%-10.5 \%}^{+18.4 \%+13 \%}$ & 1.46 & $0.648_{-17 \%-9.7 \%}^{+16.7 \%+12.3 \%}$ & 1.41 \\
2 & $0.0883_{-18.8 \%-11.9 \%}^{+19.7 \%+15.5 \%}$ & 1.47 & $0.164_{-16.5 \%-11 \%}^{+17.8 \%+14.2 \%}$ & 1.45 \\
\hline
\end{tabular}

Table 1. Cross sections for the sgluon pair production for 13 and $14 \mathrm{TeV}$ LHC as a function of the sgluon mass (see main text for more details). First error comes from the scale variation, second is the PDF uncertainty (evaluated over PDF eigenvectors using Hessian method). Relative statistical errors are below $10^{-3}$ and are not shown here. Columns $K$ give global $K$-factors. 
used: $m_{W}=80.385 \mathrm{GeV}, m_{Z}=91.1876 \mathrm{GeV}$. Top quark mass was set to $173.21 \mathrm{GeV}$ while other quarks were assumed massless in the hard matrix elements. The CKM matrix was set to identity. All samples were generated using MMTH2014nlo68cl PDFs interfaced through LHAPDF6.

\subsection{Signal}

Signal events were generated using MadGraph5_aMC@NLO v2.4.2 and an NLO-capable UFO model. For the analysis sgluon masses in the range $0.9-1.5 \mathrm{TeV}$ were considered. Renormalization and factorization scales were set equal to the sgluon mass. Sgluons were decayed into $t \bar{t}$ pairs (and further) using MadSpin [44], generating all configurations that give two same-sign muons. All spin correlations were preserved (at the LO). Total branching ratio into these channels is given by $\mathrm{BR}^{2}(W \rightarrow \mu \nu)\left(2-\mathrm{BR}^{2}(W \rightarrow \mu \nu)\right)$ where $\mathrm{BR}(W \rightarrow \mu \nu) \approx 11 \%$. Partonic events were matched to parton shower using MC@NLO [45] prescription and Pythia8 [46] v219. Pythia8 settings needed for consistent showering of MC@NLO events are described in appendix A. Since there are no genuine NLO underlying event tunes in Pythia8, the default LO tune was used.

\subsection{Background validation}

Background samples were generated using Sherpa v.2.2 [47], with virtual matrix elements provided by OpenLoops v1.3.1 [48] and evaluated using CutTools [49, 50] or COLLIER [5154]. $t \bar{t} \mu \nu_{\mu}$ events (i.e. including $\mu^{-} \bar{\nu}_{\mu}$ and $\mu^{+} \nu_{\mu}$ combinations) were generated with up to 1 additional jet at the NLO order and 3 jets at the LO, while for $t \bar{t} \mu^{+} \mu^{-}$up to 1 and 2 jets, respectively, were generated. Different multiplicities were merged/matched to parton shower using the MEPS@NLO technique $[55,56]$. In the case of $t \bar{t} \mu^{+} \mu^{-}$, a generation cut on an invariant mass of the muon pair $m_{\mu^{+} \mu^{-}}>20 \mathrm{GeV}$ was applied. Top quarks were then decayed in all ways that ensure two same-sign muons with spin correlations preserved at the LO as in the case of MadSpin. The inclusive cross sections for those samples (including appropriate top-quarks decays) are 7.77 and $5.43 \mathrm{fb}$, respectively. These predictions agree within (still very large) experimental uncertainties with the LHC measurements $[57,58]$.

The setup of Sherpa mostly follows standard settings, and only the most important ones are mentioned here. Samples were generated with EXCLUSIVE_CLUSTER_MODE $=1$ setting (meaning that only QCD splittings are considered when reconstructing parton shower history) to ensure that $t \bar{t} \mu \nu_{\mu} / t \bar{t} \mu^{+} \mu^{-}$is always identified as the core process. Since ATLAS analysis uses jets with $p_{T}>20 \mathrm{GeV}$, the merging cut was set to $15 \mathrm{GeV}$. Also, a default scale definition for the core process was used.

\section{Recasting current ATLAS $13 \mathrm{TeV}$ analysis}

The ATLAS analysis of ref. [2] targeted topologies with 2 same-sign leptons or 3 leptons, looking at 4 different signal regions. In case of the production of sgluon pair which then decays to top-quark pairs, the interesting signal region is SR3b defined in table 1 of [2]. To match experimental data as closely as possible, the detector response was parametrized using Delphes [59] v3.3.2. 
The following list gives a summary of Delphes detector card settings ${ }^{5}$ and applied cuts:

1 Muons are identified with the efficiency of $95 \%$ if they have $p_{T}>10 \mathrm{GeV}$ and $|\eta|<1.5$ and $85 \%$ if $1.5<|\eta|<2.7$. Candidate muons are required to have $p_{T}>20 \mathrm{GeV}$ and $|\eta|<2.5$. Candidate muons must also be isolated, i.e. have the scalar sum of the $p_{T}$ of tracks within a variable-size cone around the lepton, excluding its own track, less than $6 \%$ of the muon $p_{T}$. The isolation cone size is taken to be the smaller of $10 \mathrm{GeV} / p_{T}$ and 0.3 (where $p_{T}$ denotes the muon's transverse momentum). ${ }^{6}$ The analysis requires (at least) two same-sign muons fulfilling above criteria.

2 At least 3 b-tagged jets reconstructed using anti-kt algorithm [60] from Fast Jet [61, $62]$ with $p_{T}>20 \mathrm{GeV}$ and $|\eta|<2.5$ are required. Jets are $b$-tagged if they are within $\Delta R_{j b}<0.3$ of a $b$-quark which had $p_{T}^{b}>5 \mathrm{GeV}$ and $\left|\eta_{b}\right|<2.5$ with an efficiency

$$
b \text {-tagging efficient }=\frac{24 \tanh \left(0.003 \cdot p_{T}\right)}{1+0.086 \cdot p_{T}} .
$$

The probabilities of misidentifying $c$ and light quarks as $b$ ones are given by

$$
\begin{aligned}
c \text {-mistag rate } & =\frac{0.2 \tanh \left(0.02 \cdot p_{T}\right)}{1+0.0034 \cdot p_{T}}, \\
\text { light quark mistag rate } & =0.002+7.3 \cdot 10^{-6} p_{T},
\end{aligned}
$$

respectively. The efficiency formulas are based on [63]. Jet energy scale (JES) correction is applied according to the following formula ${ }^{7}$

$$
E_{j} \rightarrow \sqrt{1+(3-0.2|\eta|)^{2} / p_{T}} \cdot E_{j}
$$

$3 E_{T}^{\text {miss }}>125 \mathrm{GeV}$

4 Effective mass $m_{\text {eff }}$ of the event, defined as a scalar sum of $p_{T}$ of signal leptons, b-jets and missing $E_{T}$, must satisfy $m_{\mathrm{eff}}>650 \mathrm{GeV}$.

Table 2 shows the cross sections (in fb) for signal and selected background processes passing this sequence of cuts (cuts are stacked, i.e. a cut in the $n$-th column also implies that cuts in $n-1$ first columns were applied). Table 3 then compares final numbers of events, i.e. after multiplying last column of table 2 by $3.2 \mathrm{fb}^{-1}$ of integrated luminosity and a factor of 4 , to account for all possible leptonic channels taken into account in the ATLAS analysis, with the column SRb3 of table 5 of ref. [2]. The analysis was also validated on the signal process considered in the ATLAS work, i.e. gluino pair production with $\tilde{g} \rightarrow t \bar{t} \tilde{\chi}_{0}$ decay through offshell stops. The gluino sample was generated at the LO with up to 1 additional jet, merged using CKKW-L prescription [64] with merging scale, defined as Lund $p_{T}$, set to $300 \mathrm{GeV}$. Table 4 shows the predicted number of signal events after the considered sequence of cuts

\footnotetext{
${ }^{5}$ The complete ATLAS detector card used in this analysis can be found in supplementary materials made available together with the arXiv version of this work.

${ }^{6}$ Delphes Isolation module was modified to allow for a variable isolation cone size.

${ }^{7} \mathrm{JES}$ is applied before the requirement of $p_{T}>20 \mathrm{GeV}$.
} 


\begin{tabular}{|c|ccc|c|}
\hline & SS muon pair & $\#$ b-jets $\geq 3$ & $E_{T}^{\mathrm{miss}}>125 \mathrm{GeV}$ & $m_{\mathrm{eff}}>650 \mathrm{GeV}$ \\
\hline$t \bar{t} \mu \nu$ & 3.1876 & 0.0899 & 0.0241 & $0.0117 \pm 0.0006$ \\
$t \bar{t} \mu^{+} \mu^{-}$ & 2.850 & 0.102 & 0.0146 & $0.008 \pm 0.001$ \\
\hline$m_{O}=0.90 \mathrm{TeV}$ & 1.352 & 0.707 & 0.452 & $0.424 \pm 0.002$ \\
$m_{O}=1.00 \mathrm{TeV}$ & 0.6410 & 0.3324 & 0.2264 & $0.2172 \pm 0.0007$ \\
$m_{O}=1.25 \mathrm{TeV}$ & 0.1144 & 0.0569 & 0.0433 & $0.0426 \pm 0.0001$ \\
$m_{O}=1.50 \mathrm{TeV}$ & 0.02365 & 0.01109 & 0.00903 & $0.00897 \pm 0.00003$ \\
\hline
\end{tabular}

Table 2. Cut-flow analysis summary (numbers in fb). For brevity's sake, only errors for the final results are given. Errors are statistical only.

\begin{tabular}{|c|c|c|}
\hline & this analysis & ATLAS \\
\hline$t \bar{t} \mu \nu$ & $0.149 \pm 0.007$ & $0.10 \pm 0.05$ \\
$t \bar{t} \mu^{+} \mu^{-}$ & $0.12 \pm 0.02$ & $0.14 \pm 0.06$ \\
\hline$m_{O}=0.90 \mathrm{TeV}$ & $5.42 \pm 0.02$ & \\
$m_{O}=1.00 \mathrm{TeV}$ & $2.781 \pm 0.009$ & \\
$m_{O}=1.25 \mathrm{TeV}$ & $0.546 \pm 0.002$ & \\
$m_{O}=1.50 \mathrm{TeV}$ & $0.1148 \pm 0.0003$ & \\
\hline
\end{tabular}

Table 3. Final result of analysis (last column of table 2) after multiplying by $3.2 \mathrm{fb}^{-1}$ of integrated luminosity and roughly a factor of 4 , to account for all possible leptonic channels taken into account in the ATLAS analysis [2], compared to column SRb3 of table 5 of that analysis.

for $3.2 \mathrm{fb}^{-1}$ of integrated luminosity in comparison to the ATLAS result [65]. Contrary to the case of SM background, all possible decays of top quarks were generated. We checked on this example, that selection efficiencies calculated taking into account both electrons and muons and efficiencies calculated using only muons are roughly the same. Although electron identification criteria are tighter than the muon ones, ${ }^{8}$ the difference is within the accuracy of the simplified detector parametrization. This justifies the approach used in the case of sgluon signal and SM backgrounds, where only muonic decays were simulated.

The fact that the simplified analysis based on Delphes predicts roughly the same number of events for background coming from $t \bar{t} \mu \nu_{\mu} / t \bar{t} \mu^{+} \mu^{-}$and gluino pair production signal as the ATLAS one is a check of its implementation.

As can be seen in table 5 of ref. [2], a significant contribution to the SM background comes from processes which cannot be reliably simulated by tools like Delphes, i.e. fake/non-prompt leptons and charge flips. This implies that the cuts used in the definition of SR3b could not be changed (adapting them to the kinematics of the sgluon pair production) as it would change the size of those contributions. Therefore, to check the separating power of cuts used by ATLAS on the sgluon signal, we looked at the effective

\footnotetext{
${ }^{8}$ The selection efficiencies for both electrons and muons are taken to be the same in the Delphes card. Electron must fulfill tighter isolation criteria, though, with the isolation cone size taken to be the smaller of $10 \mathrm{GeV} / p_{T}$ and 0.2 (not 0.3 as in the case of muons). Also, candidates within the transition region between the barrel and endcap electromagnetic calorimeters, $1.37<|\eta|<1.52$, and with $|\eta|>2$ are removed.
} 


\begin{tabular}{|l|cc|}
\hline$\geq 2$ SS leptons $\left(p_{T}>20 \mathrm{GeV}\right)$ & this analysis & ATLAS \\
$\geq 3$ b-jets $\left(p_{T} \geq 20 \mathrm{GeV}\right)$ & $5.50 \pm 0.09$ & $9.28 \pm 0.18$ \\
$E_{T}^{\text {miss }}>125 \mathrm{GeV}$ & $4.34 \pm 0.06$ & $4.26 \pm 0.12$ \\
$m_{\text {eff }}>650 \mathrm{GeV}$ & $2.86 \pm 0.05$ & $3.20 \pm 0.11$ \\
\hline
\end{tabular}

Table 4. The validation of our analysis on the gluino pair production studied by ATLAS. Total number of signal events in this analysis was normalized to 275 as given by ATLAS.

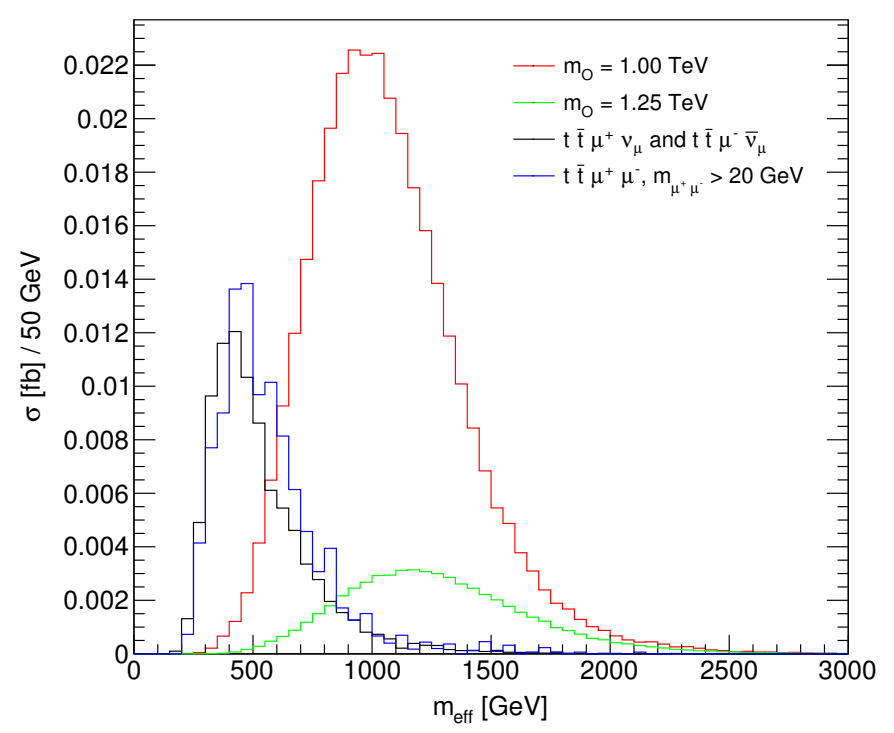

Figure 4. Effective mass spectrum after requiring 2 same-sign leptons and at least $3 b$-tagged jets (see text for details) for the signal from 1 and $1.25 \mathrm{TeV}$ sgluon pair and background from $t \bar{t} \mu^{+} \nu_{\mu} / t \bar{t} \mu^{-} \bar{\nu}_{\mu}$ and $t \bar{t} \mu^{+} \mu^{-}$.

mass spectrum after the same-sign muon pair requirement and the cut on the number of $b$-jets. This is shown in figure 4 for two sgluon masses: 1 and $1.25 \mathrm{TeV}$ and backgrounds from $t \bar{t} \mu \nu_{\mu}$ and $t \bar{t} \mu^{+} \mu^{-}$. It is clear that the cut of $m_{\mathrm{eff}}>650 \mathrm{GeV}$ used in the ATLAS analysis does also a good job of separating background from the sgluon signal.

The $95 \%$ CL ATLAS upper limit on the number of signal (BSM) events in the SR3b is 3.8. The predicted numbers of signal events for selected sgluon masses are given in table 3 . The ATLAS limit does corresponds to sgluons mass in the range $0.9<m_{O}<1 \mathrm{TeV}$. To facilitate reading of its precise value, predicted numbers of signal events are plotted in figure 5 together with the interpolation between them. From this, sgluon masses $<0.95 \mathrm{TeV}$ are excluded at $95 \% \mathrm{CL}$. This result is already on par with the $8 \mathrm{TeV}$ ATLAS exclusion, which was $1.06 \mathrm{TeV}$ for the case of a complex sgluon (i.e. with a cross section greater by a factor of 2).

The ATLAS experiment is supposed to gather $100 \mathrm{fb}^{-1}$ of integrated luminosity by the end of Run 2, which is roughly 30 times more than what is currently available. Since 


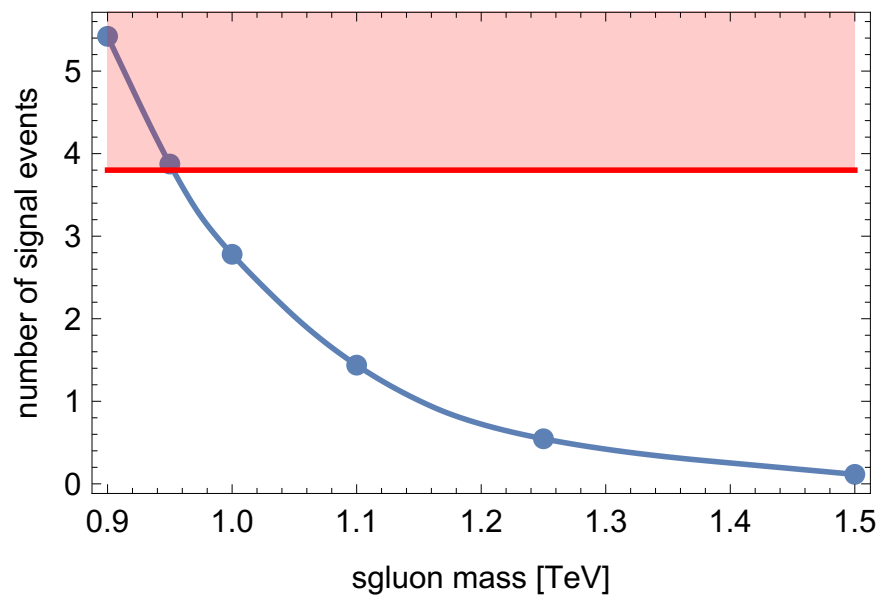

Figure 5. Predicted number of observed signal events as a function of the sgluon mass (blue points). Solid line shows interpolation between these points. Red region is excluded by ATLAS for SR3b at 95\% CL. Interpreted in the context of sgluon production, it corresponds to a lower limit on the sgluon mass $m_{O} \lesssim 0.95 \mathrm{TeV}$.

statistical significance scales like a square-root of integrated luminosity, numbers in table 3 suggest that even without further exploiting event kinematics and adapting cuts, it should be possible to exclude (or discover) sgluons with masses up to $\lesssim 1.25 \mathrm{TeV}$ by the end of Run 2.

\section{Conclusions}

In this work current ATLAS exclusion limits coming from the search of 4-top quark final state in events with same-sign leptons were recast to the case of sgluon pair production. Although sgluons decay to a top-quark pair without the presence of the invisible LSP assumed in the ATLAS analysis, the cuts used prove to work well also in this case. Data sample of $3.2 / \mathrm{fb}$ allows to exclude sgluons with masses $\lesssim 0.95 \mathrm{TeV}$, a result already on par with the $8 \mathrm{TeV}$ exclusion. It should, therefore, be possible to push this limit up to $1.25 \mathrm{TeV}$ by the end of Run 2 based just on the increased statistics. Naturally, with an increased statistics, experimental collaboration will be able to adapt the selection criteria to further exploit sgluon kinematics, pushing this exclusion even further. We therefore encourage experimentalist to look into this.

\section{Acknowledgments}

I thank Frank Siegert for his help concerning Sherpa, Stefan Prestel for his constant support on Pythia8, Dominik Stöckinger and Philip Diessner for insightful discussions on NLO (S)QCD corrections and Jan Kalinowski for suggesting the topic of this work. Work supported in part by the German DFG grant STO 876/4-1 and the National Science Centre, Poland, the HARMONIA project under contract UMO2015/18/M/ST2/00518 (2016-2019). 


\section{A Pythia8 technical setup}

By default, the final state shower algorithm in Pythia8 is based on dipole-style recoils. As stated in Pythia8 manual, for MC@NLO, where a full analytic knowledge of the shower radiation pattern is needed, one has to switch to global recoil approach which does not contain color coherence phenomena (and hence factorizes). A minimal set of settings needed to consistently shower MC@NLO events is then given by ${ }^{9}$

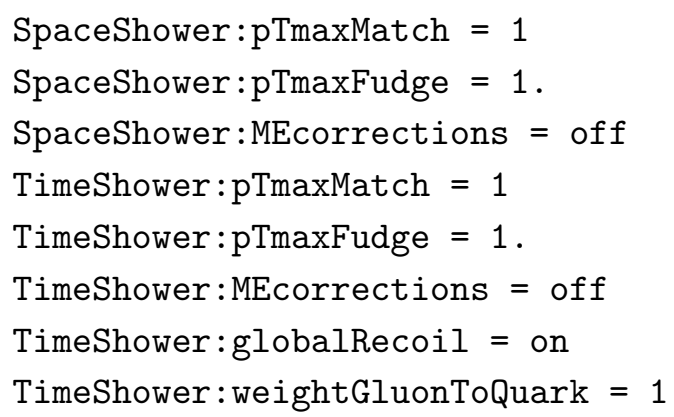

Those settings cannot be modified. What can be chosen, though, is when to return from the global recoil mode to the dipole recoil. Since color coherence phenomena are very important (see for example [67]), it is advantageous to switch back to dipole recoils already after the first emission. This can be done in two ways, either by setting TimeShower:globalRecoilMode $=1$ or by setting it to 2 . Option 2 applies global recoil only if the first branching in evolution is a timelike splitting of a parton in an event with Born-like kinematics (the so called $\mathbb{S}$-events in the MC@NLO language), while for option 1 this is done both for Born-like $(\mathbb{S})$ and real-emission events ( $\mathbb{H}$-events). With option 2, the impact of global recoil should be minimal. For options 1 and 2, a maximal number of splittings in the timelike shower with global recoil strategy should be set to 1 through TimeShower:nMaxGlobalBranch flag. Also, to distinguish between $\mathbb{S}$ and $\mathbb{H}$ events, the number of color-charged particles for Born-like configurations must be given through TimeShower:nPartonsInBorn option. The MC@NLO matching is done at the level of the hard process. To that end, Pythia8 removes decay chains generated by MadSpin by traversing the event tree and identifying intermediate particles with status code ISTUP $= \pm 2[68]$ which have a single parent. TimeShower:nPartonsInBorn then counts the number of remaining color-charged particles. For the sgluon pair production one therefore sets:

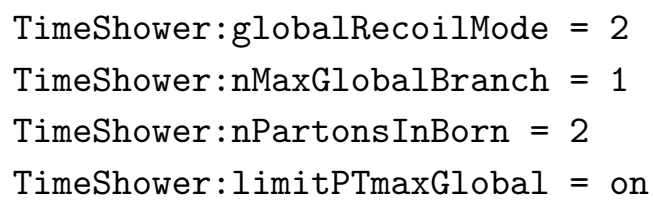

Open Access. This article is distributed under the terms of the Creative Commons Attribution License (CC-BY 4.0), which permits any use, distribution and reproduction in any medium, provided the original author(s) and source are credited.

\footnotetext{
${ }^{9}$ See Pythia8 manual at http://home.thep.lu.se/ torbjorn/pythia82html/Welcome.html, section Link to Other Programs $\rightarrow$ Matching and Merging $\rightarrow$ aMC@NLO Matching. See also the discussion in ref. [66].
} 


\section{References}

[1] M. Heikinheimo, M. Kellerstein and V. Sanz, How Many Supersymmetries?, JHEP 04 (2012) 043 [arXiv:1111.4322] [INSPIRE].

[2] ATLAS collaboration, Search for supersymmetry at $\sqrt{s}=13$ TeV in final states with jets and two same-sign leptons or three leptons with the ATLAS detector, Eur. Phys. J. C 76 (2016) 259 [arXiv: 1602.09058] [INSPIRE].

[3] ATLAS collaboration, Search for new physics using events with b-jets and a pair of same charge leptons in 3.2 $\mathrm{fb}^{-1}$ of $\mathrm{pp}$ collisions at $\sqrt{\mathrm{s}}=13$ TeV with the ATLAS detector, ATLAS-CONF-2016-032 (2016).

[4] ATLAS collaboration, Search for four-top-quark production in final states with one charged lepton and multiple jets using 3.2 $\mathrm{fb}^{-1}$ of proton-proton collisions at $\sqrt{\mathrm{s}}=13$ TeV with the ATLAS detector at the LHC, ATLAS-CONF-2016-020 (2016).

[5] ATLAS collaboration, Search for production of vector-like top quark pairs and of four top quarks in the lepton-plus-jets final state in pp collisions at $\sqrt{s}=13$ TeV with the ATLAS detector, ATLAS-CONF-2016-013 (2016).

[6] CMS collaboration, Search for new physics in same-sign dilepton events in proton-proton collisions at $\sqrt{s}=13$ TeV, Eur. Phys. J. C 76 (2016) 439 [arXiv:1605.03171] [INSPIRE].

[7] CMS collaboration, Search for supersymmetry in pp collisions at $\sqrt{s}=13$ TeV in the single-lepton final state using the sum of masses of large-radius jets, JHEP 08 (2016) 122 [arXiv: 1605.04608] [INSPIRE].

[8] CMS collaboration, Search for supersymmetry in the multijet and missing transverse momentum final state in pp collisions at 13 TeV, Phys. Lett. B 758 (2016) 152 [arXiv: 1602.06581] [INSPIRE].

[9] S.Y. Choi, M. Drees, J. Kalinowski, J.M. Kim, E. Popenda and P.M. Zerwas, Color-Octet Scalars of $N=2$ Supersymmetry at the LHC, Phys. Lett. B 672 (2009) 246 [arXiv:0812.3586] [INSPIRE].

[10] K. Benakli, L. Darmé, M.D. Goodsell and J. Harz, The Di-Photon Excess in a Perturbative SUSY Model, Nucl. Phys. B 911 (2016) 127 [arXiv: 1605.05313] [InSPIRE].

[11] T. Plehn and T.M.P. Tait, Seeking Sgluons, J. Phys. G 36 (2009) 075001 [arXiv:0810.3919] [INSPIRE].

[12] S. Calvet, B. Fuks, P. Gris and L. Valery, Searching for sgluons in multitop events at a center-of-mass energy of $8 \mathrm{TeV}$, JHEP 04 (2013) 043 [arXiv:1212.3360] [INSPIRE].

[13] C. Kilic, S. Schumann and M. Son, Searching for Multijet Resonances at the LHC, JHEP 04 (2009) 128 [arXiv:0810.5542] [INSPIRE].

[14] S. Schumann, A. Renaud and D. Zerwas, Hadronically decaying color-adjoint scalars at the LHC, JHEP 09 (2011) 074 [arXiv:1108.2957] [INSPIRE].

[15] W. Kotlarski, A. Kalinowski and J. Kalinowski, Searching for Sgluons in the Same-sign Leptons Final State at the LHC, Acta Phys. Polon. B 44 (2013) 2149.

[16] C.-Y. Chen, A. Freitas, T. Han and K.S.M. Lee, Heavy Color-Octet Particles at the LHC, JHEP 05 (2015) 135 [arXiv: 1410.8113] [INSPIRE].

[17] L. Beck, F. Blekman, D. Dobur, B. Fuks, J. Keaveney and K. Mawatari, Probing top-philic sgluons with LHC Run I data, Phys. Lett. B 746 (2015) 48 [arXiv:1501.07580] [InSPIRE]. 
[18] B.A. Dobrescu, K. Kong and R. Mahbubani, Massive color-octet bosons and pairs of resonances at hadron colliders, Phys. Lett. B 670 (2008) 119 [arXiv:0709.2378] [InSPIRE].

[19] C. Kilic and T. Okui, The LHC Phenomenology of Vectorlike Confinement, JHEP 04 (2010) 128 [arXiv: 1001.4526] [INSPIRE].

[20] G. Burdman, B.A. Dobrescu and E. Ponton, Resonances from two universal extra dimensions, Phys. Rev. D 74 (2006) 075008 [hep-ph/0601186] [INSPIRE].

[21] C. Degrande, B. Fuks, V. Hirschi, J. Proudom and H.-S. Shao, Automated next-to-leading order predictions for new physics at the LHC: the case of colored scalar pair production, Phys. Rev. D 91 (2015) 094005 [arXiv: 1412.5589] [InSPIRE].

[22] G.D. Kribs, E. Poppitz and N. Weiner, Flavor in supersymmetry with an extended R-symmetry, Phys. Rev. D 78 (2008) 055010 [arXiv:0712.2039] [INSPIRE].

[23] L. Randall and N. Rius, The Minimal U(1)-R symmetric model revisited, Phys. Lett. B 286 (1992) 299 [INSPIRE].

[24] P. Dießner, J. Kalinowski, W. Kotlarski and D. Stöckinger, Higgs boson mass and electroweak observables in the MRSSM, JHEP 12 (2014) 124 [arXiv:1410.4791] [INSPIRE].

[25] P. Diessner, J. Kalinowski, W. Kotlarski and D. Stöckinger, Two-loop correction to the Higgs boson mass in the MRSSM, Adv. High Energy Phys. 2015 (2015) 760729 [arXiv: 1504.05386] [INSPIRE].

[26] P. Diessner, J. Kalinowski, W. Kotlarski and D. Stöckinger, Exploring the Higgs sector of the MRSSM with a light scalar, JHEP 03 (2016) 007 [arXiv: 1511.09334] [INSPIRE].

[27] J. Ellis, J. Quevillon and V. Sanz, Doubling Up on Supersymmetry in the Higgs Sector, JHEP 10 (2016) 086 [arXiv: 1607.05541] [INSPIRE].

[28] J. Braathen, M.D. Goodsell and P. Slavich, Leading two-loop corrections to the Higgs boson masses in SUSY models with Dirac gauginos, JHEP 09 (2016) 045 [arXiv: 1606.09213] [INSPIRE].

[29] E. Bertuzzo, C. Frugiuele, T. Gregoire and E. Ponton, Dirac gauginos, $R$ symmetry and the $125 \mathrm{GeV}$ Higgs, JHEP 04 (2015) 089 [arXiv: 1402.5432] [INSPIRE].

[30] ATLAS collaboration, Search for pair-produced massive coloured scalars in four-jet final states with the ATLAS detector in proton-proton collisions at $\sqrt{s}=7 \mathrm{TeV}$, Eur. Phys. J. C 73 (2013) 2263 [arXiv: 1210.4826] [INSPIRE].

[31] ATLAS collaboration, Search for production of vector-like quark pairs and of four top quarks in the lepton-plus-jets final state in pp collisions at $\sqrt{s}=8 \mathrm{TeV}$ with the ATLAS detector, JHEP 08 (2015) 105 [arXiv: 1505.04306] [INSPIRE].

[32] D. Goncalves-Netto, D. Lopez-Val, K. Mawatari, T. Plehn and I. Wigmore, Sgluon Pair Production to Next-to-Leading Order, Phys. Rev. D 85 (2012) 114024 [arXiv:1203.6358] [INSPIRE].

[33] C. Degrande, C. Duhr, B. Fuks, D. Grellscheid, O. Mattelaer and T. Reiter, UFO - The Universal FeynRules Output, Comput. Phys. Commun. 183 (2012) 1201 [arXiv:1108.2040] [INSPIRE].

[34] J. Alwall et al., The automated computation of tree-level and next-to-leading order differential cross sections and their matching to parton shower simulations, JHEP 07 (2014) 079 [arXiv: 1405.0301] [INSPIRE]. 
[35] A. Alloul, N.D. Christensen, C. Degrande, C. Duhr and B. Fuks, FeynRules 2.0 - A complete toolbox for tree-level phenomenology, Comput. Phys. Commun. 185 (2014) 2250 [arXiv: 1310.1921] [INSPIRE].

[36] C. Degrande, Automatic evaluation of UV and R2 terms for beyond the Standard Model Lagrangians: a proof-of-principle, Comput. Phys. Commun. 197 (2015) 239 [arXiv:1406.3030] [INSPIRE].

[37] T. Hahn, Generating Feynman diagrams and amplitudes with FeynArts 3, Comput. Phys. Commun. 140 (2001) 418 [hep-ph/0012260] [INSPIRE].

[38] T. Hahn and M. Pérez-Victoria, Automatized one loop calculations in four-dimensions and D-dimensions, Comput. Phys. Commun. 118 (1999) 153 [hep-ph/9807565] [InSPIRE].

[39] https://feynrules.irmp.ucl.ac.be/wiki/NLOModels, [accessed November 4, 2016].

[40] L.A. Harland-Lang, A.D. Martin, P. Motylinski and R.S. Thorne, Parton distributions in the LHC era: MMHT 2014 PDFs, Eur. Phys. J. C 75 (2015) 204 [arXiv: 1412.3989] [InSPIRE].

[41] A. Buckley et al., LHAPDF6: parton density access in the LHC precision era, Eur. Phys. J. C 75 (2015) 132 [arXiv: 1412.7420] [INSPIRE].

[42] B.W. Harris and J.F. Owens, The two cutoff phase space slicing method, Phys. Rev. D 65 (2002) 094032 [hep-ph/0102128] [INSPIRE].

[43] W.M. Kotlarski, Analysis of the R-symmetric supersymmetric models including quantum corrections, arXiv: 1611.06622 [INSPIRE].

[44] P. Artoisenet, R. Frederix, O. Mattelaer and R. Rietkerk, Automatic spin-entangled decays of heavy resonances in Monte Carlo simulations, JHEP 03 (2013) 015 [arXiv:1212.3460] [INSPIRE].

[45] S. Frixione and B.R. Webber, Matching NLO QCD computations and parton shower simulations, JHEP 06 (2002) 029 [hep-ph/0204244] [INSPIRE].

[46] T. Sjöstrand et al., An introduction to PYTHIA 8.2, Comput. Phys. Commun. 191 (2015) 159 [arXiv: 1410.3012] [INSPIRE].

[47] T. Gleisberg et al., Event generation with SHERPA 1.1, JHEP 02 (2009) 007 [arXiv:0811.4622] [INSPIRE].

[48] F. Cascioli, P. Maierhofer and S. Pozzorini, Scattering Amplitudes with Open Loops, Phys. Rev. Lett. 108 (2012) 111601 [arXiv:1111.5206] [INSPIRE].

[49] G. Ossola, C.G. Papadopoulos and R. Pittau, CutTools: A program implementing the OPP reduction method to compute one-loop amplitudes, JHEP 03 (2008) 042 [arXiv:0711.3596] [INSPIRE].

[50] G. Ossola, C.G. Papadopoulos and R. Pittau, Reducing full one-loop amplitudes to scalar integrals at the integrand level, Nucl. Phys. B 763 (2007) 147 [hep-ph/0609007] [INSPIRE].

[51] A. Denner and S. Dittmaier, Scalar one-loop 4-point integrals, Nucl. Phys. B 844 (2011) 199 [arXiv: 1005.2076] [INSPIRE].

[52] A. Denner and S. Dittmaier, Reduction schemes for one-loop tensor integrals, Nucl. Phys. B 734 (2006) 62 [hep-ph/0509141] [INSPIRE].

[53] A. Denner, S. Dittmaier and L. Hofer, Collier: a fortran-based Complex One-Loop LIbrary in Extended Regularizations, Comput. Phys. Commun. 212 (2017) 220 [arXiv:1604.06792] [INSPIRE]. 
[54] A. Denner and S. Dittmaier, Reduction of one loop tensor five point integrals, Nucl. Phys. B 658 (2003) 175 [hep-ph/0212259] [INSPIRE].

[55] S. Hoeche, F. Krauss, M. Schonherr and F. Siegert, QCD matrix elements + parton showers: The NLO case, JHEP 04 (2013) 027 [arXiv:1207.5030] [INSPIRE].

[56] T. Gehrmann, S. Hoche, F. Krauss, M. Schonherr and F. Siegert, NLO QCD matrix elements + parton showers in $e^{+} e^{-} \rightarrow$ hadrons, JHEP 01 (2013) 144 [arXiv:1207.5031] [INSPIRE].

[57] ATLAS collaboration, Measurement of the $t \bar{t} Z$ and $t \bar{t} W$ production cross sections in multilepton final states using 3.2 $\mathrm{fb}^{-1}$ of pp collisions at $13 \mathrm{TeV}$ at the $\mathrm{LHC}$, ATLAS-CONF-2016-003 (2016).

[58] CMS collaboration, Measurement of the cross section of top quark pair production in association with a $Z$ boson in pp collisions at 13 TeV, CMS-PAS-TOP-16-009 (2016).

[59] DELPHES 3 collaboration, J. de Favereau et al., DELPHES 3, A modular framework for fast simulation of a generic collider experiment, JHEP 02 (2014) 057 [arXiv:1307.6346] [INSPIRE].

[60] M. Cacciari, G.P. Salam and G. Soyez, The anti-k(t) jet clustering algorithm, JHEP 04 (2008) 063 [arXiv:0802.1189] [INSPIRE].

[61] M. Cacciari, G.P. Salam and G. Soyez, FastJet User Manual, Eur. Phys. J. C 72 (2012) 1896 [arXiv: 1111.6097] [INSPIRE].

[62] M. Cacciari and G.P. Salam, Dispelling the $N^{3}$ myth for the $k_{t}$ jet-finder, Phys. Lett. B 641 (2006) 57 [hep-ph/0512210] [INSPIRE].

[63] ATLAS collaboration, Expected performance of the ATLAS b-tagging algorithms in Run-2, ATL-PHYS-PUB-2015-022 (2015).

[64] L. Lönnblad and S. Prestel, Matching Tree-Level Matrix Elements with Interleaved Showers, JHEP 03 (2012) 019 [arXiv: 1109.4829] [INSPIRE].

[65] https://atlas.web.cern.ch/Atlas/GROUPS/PHYSICS/PAPERS/SUSY-2015-09/ tabaux_03.pdf [accessed on 26.11.2016].

[66] ATLAS collaboration, Modelling of the $t \bar{t} H$ and $t \bar{t} V(V=W, Z)$ processes for $\sqrt{s}=13 \mathrm{TeV}$ ATLAS analyses, ATL-PHYS-PUB-2016-005 (2016).

[67] CMS collaboration, Probing color coherence effects in pp collisions at $\sqrt{s}=7 \mathrm{TeV}$, Eur. Phys. J. C 74 (2014) 2901 [arXiv:1311.5815] [INSPIRE].

[68] E. Boos et al., Generic user process interface for event generators, hep-ph/0109068 [INSPIRE]. 